\title{
Erratum: Activating ATR, the devil's in the dETAA1I
}

\section{Wojciech Niedzwiedz}

Nature Cell Biology 18, 1120-1122 (2016); published online 27 October 2016; corrected after print 4 November 2016.

In the version of this News and Views originally published, there was an error in Fig. 2: the protein 'TOPBP1' should have been in contact with the protein 'ATR'. This has been corrected in the online versions of the News and Views. 